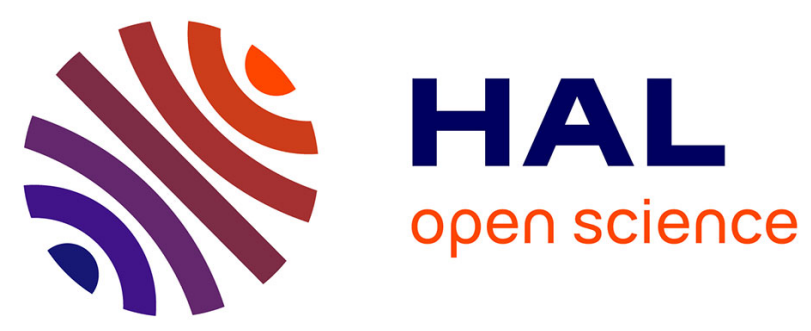

\title{
Baseband Fading Channel Simulator for Inter-Vehicle Communication using SystemC-AMS
}

Abdelbasset Massouri, Antoine Lévêque, Laurent Clavier, Michel Vasilevski, Andreas Kaiser, Marie-Minerve Louerat

\section{To cite this version:}

Abdelbasset Massouri, Antoine Lévêque, Laurent Clavier, Michel Vasilevski, Andreas Kaiser, et al.. Baseband Fading Channel Simulator for Inter-Vehicle Communication using SystemC-AMS. 2010 IEEE International Behavioral Modeling and Simulation Conference (BMAS 2010), Sep 2010, San Jose, CA, United States. pp.36-41. hal-00632156

\section{HAL Id: hal-00632156 https://hal.science/hal-00632156}

Submitted on 13 Oct 2011

HAL is a multi-disciplinary open access archive for the deposit and dissemination of scientific research documents, whether they are published or not. The documents may come from teaching and research institutions in France or abroad, or from public or private research centers.
L'archive ouverte pluridisciplinaire HAL, est destinée au dépôt et à la diffusion de documents scientifiques de niveau recherche, publiés ou non, émanant des établissements d'enseignement et de recherche français ou étrangers, des laboratoires publics ou privés. 


\title{
Baseband Fading Channel Simulator for Inter-Vehicle Communication using SystemC-AMS
}

\author{
Abdelbasset Massouri $^{1}$, Antoine Lévêque ${ }^{2}$, Laurent Clavier $^{1}$, Michel Vasilevski ${ }^{2}$, \\ Andreas Kaiser ${ }^{1}$, Marie-Minerve Louërat ${ }^{2}$ \\ Institut d'Electronique, de Microlectronique et de Nanotechnologie ${ }^{1}$, University Pierre \& Marie Curie, LIP6 ${ }^{2}$
}

\begin{abstract}
System level modeling and simulation have become a key issue in analyzing, optimizing and designing wireless systems. In this paper, modeling RF front end devices and radio propagation channel especially fading time-variant channel for InterVehicle Communication using SystemC-AMS are investigated. Discrete baseband wireless channel model based on Tapped Delay Line model is presented. Time-variant Rayleigh and Rician fading are implemented in SystemC-AMS. The capabilities of SystemCAMS are tested and compared to matlab for five vehicles scenario. With this framework, several criteria such as performance and optimal value of the system parameters of a WSN node can be evaluated and predicted before any hardware and software design.
\end{abstract}

Index Terms-WSN, Inter-Vehicle Communication, SystemCAMS, base band modeling, wireless channel, TDMA.

\section{INTRODUCTION}

Modern wireless communication systems are highly complex to design. The complexity continues to increase due to advanced modulation schemes, multiple protocols and standards, miniaturization and power consumption constraints, as well as additional functionality such as color display, cameras and embedded applications. During this last decade, all the digital, analog, mixed-signal and radio-frequency (RF) components of a wireless communication system are integrated into a single integrated circuit (chip) named System-on-chip (SoC). At the same time both silicon area - which means costs and power consumption of the devices have to be reduced and the design time shortened. This conflictive demands force the need for efficient design and verification methods. To have short and reliable design cycles, verification is necessary very early in the design process. Modeling and simulation need to support the design steps from the specification to the overall system verification in order to bridge the gaps between system specification, system simulation, and circuit level simulation. One of the application that uses wireless communication systems is Wireless Sensor Network (WSN). A WSN consists of several wireless sensor nodes placed regularly or irregularly. WSNs have critical applications in science, medicine, commerce and road traffic. In this work we consider WSN for Inter-Vehicle communication. A scenario of 5 vehicles is considered. Each vehicle is equipped with a wireless sensor node. In the presented work we consider the data communication. We encounter two main difficulties on this system simulation: the nonlinearity of the RF devices and the wideband time-varying channel. Besides, we also have to consider the broadcast characteristics of radio propagation.
This means that the transmitted signal is received by all active receivers through different channels. Consequently, in our scenario we have 5 communicating nodes linked by 20 different radio channels.

The rest of the paper is organized as follows: Section II describes the application and the simulation platform. In Section III the radio propagation channel modeling is presented and its SystemC-AMS implementation is explained. Finally section IV presents and discusses the results.

\section{APPLICATION AND SIMULATION PLATFORM}

The application is a complete heterogeneous system consisting of 5 cars communicating with each other. Each car is equipped with a wireless sensor. The nodes are asssumed synchronous in the current application since they share the same real time clock. The cars are identical from the hardware and software point of view. Each car has red back lights. They are all following each other along a straight road. An imaging sensor (digital camera) in the front of each car captures the back red lights of the front car and sends the digitized image to the processor of the node. The image is processed by the node processor to estimate the relative distance between two successive cars. Each car has an RF transceiver which sends a message (car identifier, relative distance with front car) through the channel and receives the similar information from the other cars, using the TDMA protocol. The processor inside the sensor can therefore compute and estimate the relative position of the other cars. The RF transmission channel is perturbed to take into account attenuation and multipath propagation. The heterogeneous models involved by this application belong to the digital, analog, RF and optical domain.

Figure 1 presents the simulation platform with 5 cars communicating through a wireless channel. This platform is composed of three main parts:

1) stimulus: it is used especially to initialize the scenario with the starting position of each vehicle. It provides positions and speeds of vehicles to the communication channels in order to calculate the attenuation and the Doppler shift.

2) Communicating Nodes (or Vehicles): the main functions performed by the RF transceiver of the considered nodes are:

- QPSK modulation

- Shape filtering at the transmitter and adaptive filtering at the receiver. The considered filter is a Root 


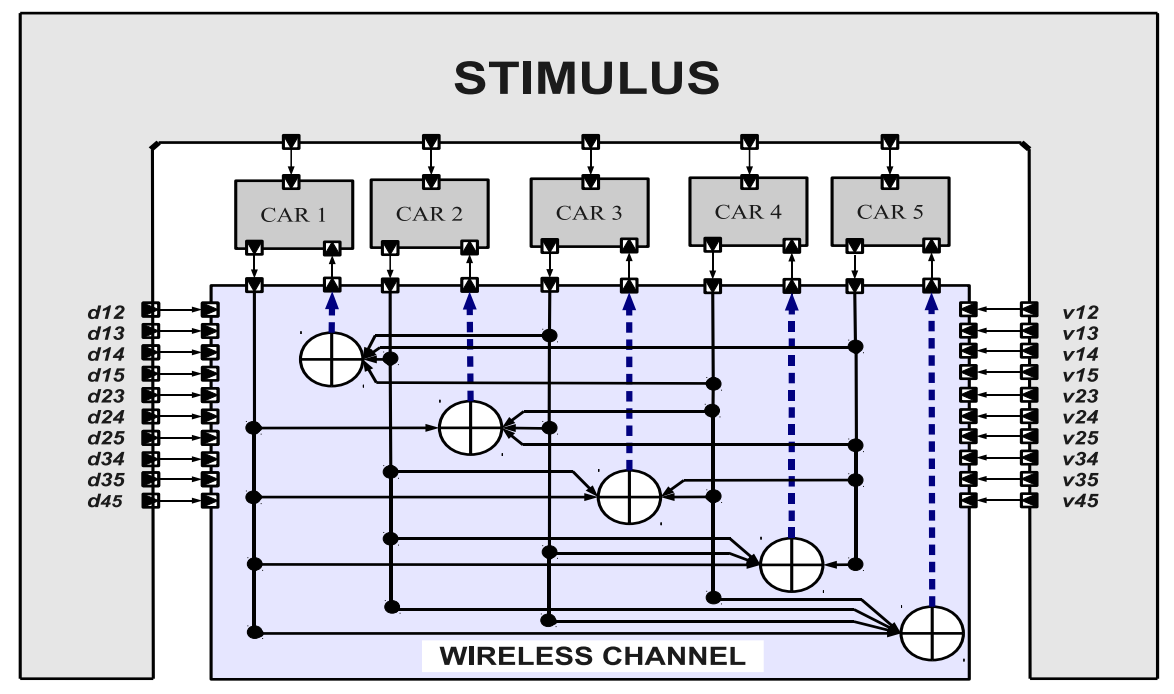

Figure 1. Simulation Platform for a WSN of 5 Nodes.

Raised Cosine Filter because it avoids the Inter Symbol Interference. The parameters of this filter are the roll-off factor $(0.5)$, its length (8) and the number of samples per symbol (16).

- Application of the non-linearities introduced by RF devices such as mixer and power amplifier at the transmitter and the low noise amplifier and mixer at the receiver. The nonlinearity models are polynomial static models.

Depending on the time slot, one car is acting as the transmitter and the other ones as the four receivers.

3) Wireless Channels: the five source nodes are connected to the five destination nodes through 20 radio channels. Figure 1 shows that each car receives the complex sum of the four transmitted signals from the other cars. This sum allows to take into consideration the multiaccess interference between the communicating nodes. The 20 additional entries convey positions and speeds from the stimulus to the radio channels. Each radio channel consists of pathloss, multipath, and additive white gaussian noise. The multipath contribution is a FIR filter whose order is equal to the number of paths (Ntaps). For each source sample transmitted through channel, we proceed as follows:

- based on speeds we compute the IIR doppler filter

- generate Ntaps independant complex gaussian random variables

- those values go through Ntaps IIR filter to obtain the time-varying channel coefficients.

- the source sample is filtered by the TDL filter to simulate the channel crossing.

- necessary datas are stored before processing the next source sample.

Such a system level simulation is very complex and time consuming. This computational complexity is not only due to the functions performed by the 5 nodes, but also to the complexity of the time-varying wireless channels.

Although the final goal is the complete simulation of the sensor functionalities (sensing, transmitting and receiving), we present in this paper only the communication part which is already a difficult challenge when several nodes are considered.

The recently standardized language SystemC AMS extensions [1], [2] has been used to model the platform of Fig. 1 together with the proof-of-concept simulator provided by Fraunhofer Institute [3]. The SystemC AMS extensions are built on top of the SystemC language standard to model heterogeneous systems. Different modeling formalism are defined by the standard to model heterogeneous system-level at different levels of abstraction. The modeling formalism used in this work is the Timed Data Flow (TDF). TDF Model of Computation (MoC) is used to describe complex non-conservative discrete-time behavior. It is based on the Synchronous Data Flow (SDF) formalism. Unlike the untimed SDF model of computation, TDF is a discrete-time modeling style which considers data as sampled signals. These signals are updated at discrete points in time and propagate discrete or continuous values.

A TDF model consists of a set of interconnected TDF modules which create a directed graph called TDF cluster. TDF modules are the nodes of the graph and the signals are the edges of the graph. A TDF module may have several input and output ports. TDF signals are used to connect ports of different modules. Each TDF module contains a $\mathrm{C}++$ method called processing() which computes a mathematical function depending only on its input and possible internal state. A given function is computed if and only if there are enough samples available at the input ports. In this case, the samples computed by the function are written to the convenient output ports. Provided that the time step data assigned by the designer on the ports and modules of a TDF cluster are compatible, the order and the number of read and written samples can be 
determined before starting the simulation and a static schedule of the cluster can be derived. This static schedule speeds up the simulation over event-driven simulation kernels.

\section{WIRELESS CHANNEL MODEL}

In this section the wireless propagation phenomenon is presented. Due to the limited space, we will not describe $\mathrm{RF}$ modules and nonlinearities that are polynomial functions but we only focus on the time varying channel. Since the framework proposed by Bello [4], many works have been done on channel modeling. To represent the main physical phenomenons of wave propagation the channel effect is divided in three parts: mean attenuation at a given distance, large scale fading (shadowing) due to the environment, small scale fading due to multipath. In this paper we only consider the first and third effect. Large scale fading is a slowly varying effect and has a little impact on the computational burden of the channel simulation. The time varying multipath contribution is the most difficult part to implement and we will essentially detail it.

\section{A. Pathloss}

Path loss is defined as the difference between the received and transmitted powers and depends on the distance between them. The path loss $L_{d B}$ can be expressed (in $d B$ ), with a distance measured in $\mathrm{km}$ and the carrier frequency in $\mathrm{MHz}$, by:

$$
L_{d B}=32.4+10 \gamma \log \left(d_{k m}\right)+20 \log \left(f_{M H z}\right) .
$$

The environment effect is resumed in the attenuation coefficient $\gamma$. An additional random variable - generally assumed lognormal - can be added to represent the effect of large scale fading.

\section{B. Small Scale Fading}

Several versions of the transmitted signal $s(t)$ sum up at the destination node to result in the received signal $r(t)$ :

$$
r(t)=\sum_{k=0}^{K(t)-1} \beta_{k}(t) \cdot e^{j \cdot \phi_{k}(t)} \cdot s\left(t-\tau_{k}(t)\right) .
$$

Variables $\beta_{k}(t), \phi_{k}(t)$ and $\tau_{k}(t)$ are, respectively, the amplitude, the phase and the time of arrival of the $k^{t h}$ ray and $K(t)$ is the time-varying number of multipaths. The channel contribution can be represented by the popular tapped-delayline (TDL) model [8], [11]. An equivalent representation of the baseband channel can be done using uniformly spaced tap coefficients, only considering delays that are multiples of the sampling time $T_{s}$. We can also assume that the number of discrete components is time-invariant $(K(t)=K)$, which leads to the impulse response of the channel:

$$
\tilde{c}(\tau, t)=\sum_{k=0}^{K-1} \tilde{g}_{k}(t) \delta\left(\tau-k T_{s}\right),
$$

where $\tilde{g}_{k}(t)$ is a complex variable that can be deduced from the channel coefficients and the sampling time. This model has been successfully used in several situations (for example GSM or UMTS) and is an accurate representation of the real channel as long as the transmission bandwidth is not too large. Indeed, this models becomes less convenient for Ultra Wide Band channel. A representation of the channel is given in Fig. 2.

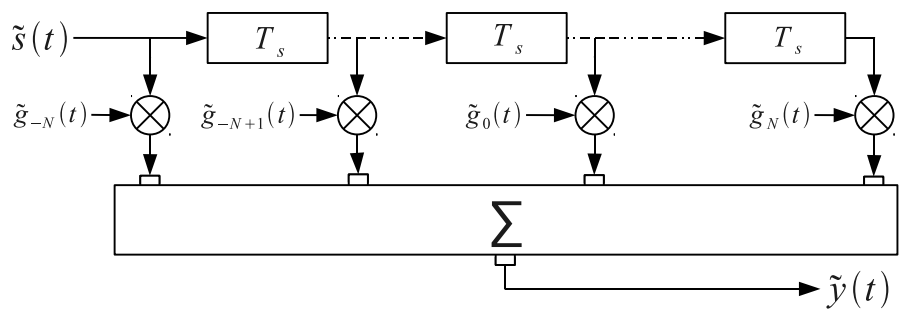

Figure 2. Bandlimited TDL channel model with equal spacings.

1) Tap coefficients' time evolution: the tap coefficients are independent random processes. Their amplitude distribution is generally assumed to be Rayleigh or Rice [9], which means that the $\tilde{g}_{k}(t)$ are complex Gaussian. Their spectrum is called the Doppler power spectrum. While different $\tilde{g}_{k}(t)$ can have distinct Doppler power spectra (see for example the COST 207 channel model [10]), for clarity and simplicity we assume the same Doppler spectrum for each coefficient, i.e. $S_{k}(f)=S(f), k=0,1, \ldots K-1$. A methodology to simulate the Rayleigh or Rice fading channel consists in shaping a complex Gaussian white noise source by the appropriate Doppler spectrum. The filtered Gaussian noise (FGN) approach can be summarized as follows, for each fading process:

- Generate a complex Gaussian process with variance 1.

- Filter the process with a Doppler filter with a frequency response $H(f)=\sqrt{S(f)}$ and impulse response $h(t)=$ $F^{-1}(H(f))$, where $F^{-1}(\cdot)$ denotes inverse Fourier transform. The impulse response is sampled at a rate $f_{s}$, to obtain a discrete-time impulse response $h[n]$.

The two steps are detailed in the following paragraphs.

a) Complex Gaussian Noise Generation: To create Complex Gaussian variable we generate 2 real independant Gaussian variables using the Box-Müller method [5].

b) Doppler Filtering: The purpose of the Doppler filter is to shape the generated complex Gaussian noise process such that the filtered process has the desired Doppler power spectrum. Several spectra models are used to model fading process. Since the subject of this work is a particular case of WSN (inter-vehicle communication) Jakes power spectrum (PSD) [6] is an appropriate choice. The baseband normalized Jakes Doppler spectrum is given analytically by:

$$
S(f)=\frac{1}{\pi f_{d} \sqrt{1-\left(f / f_{d}\right)^{2}}},|f| \leqslant f_{d},
$$

where $f_{d}$ is the maximum Doppler shift. The time variant channel is generated by filtering the complex gaussian coefficients of the channel with a filter whose transfer function is $|H(f)|=\sqrt{S(f)}$. One way to implement this filter is in the 
frequency domain [12], [13], then using an inverse Fourier transform (IFFT) to obtain the tap coefficients in the time domain. One major disadvantage of the IFFT method is its block-oriented nature, which requires all channel coefficients to be generated and stored before the data are sent through the channel. This implies significant memory requirements and may preclude unbounded continuous transmission, which is usually preferred in long-running applications. An alternative technique is to filter the time-domain Gaussian in-phase and quadrature components with the Doppler filter (see Fig. 3).

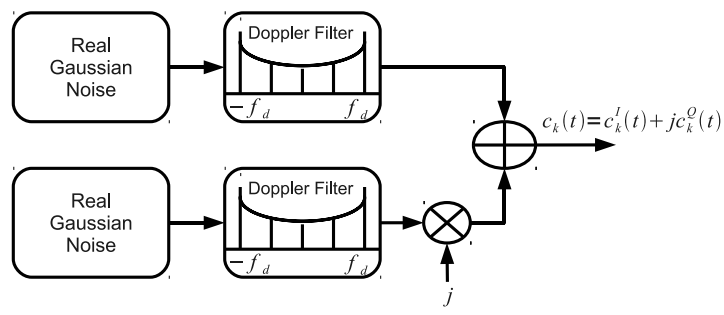

Figure 3. One coefficient Rayleigh fading generator.

The reciprocal square root of the Jakes' PSD is an irrational function [15], which cannot be exactly implemented, and thus, it is common to use a rational approximation. To provide spectral shaping for a rational implementation, we use transformation-based filter designs [18], [19]. An important filter design constraint to consider is that in wireless commu- ${ }_{10}^{9}$ nication channels, $f_{d}<<1 / T s$. Consequently, the bandwidth 11 of the spectral shaping filter must typically be much narrower ${ }_{13}^{12}$ than the bandwidth of the transmitted signal. For example, 14 consider a system compliant to the IEEE standard for vehicular 15 communications (802.11p or WAVE as Wireless Access in Ve- 17 hicular Environments). It operates at $f_{c}=5.9 \mathrm{GHz}$, and it has 18 $10 \mathrm{MHz}$ of bandwidth. If the mobile receiver has a maximum 20 speed of $v=120 \mathrm{~km} / \mathrm{h}$ relative to the transmitter, then the ${ }_{22}^{21}$ Doppler shift is $f_{d}=f_{c}(v / c)=500 \mathrm{~Hz}$, where $c$ is the 23 speed of light. If the signal is sampled at $f_{s}=160 \mathrm{MHz}$, then ${ }_{25}^{24}$ the normalized Doppler frequency would be $f_{d} / f_{s} \approx 4.10^{-6}$. 26 The design of extremely narrowband digital filter may run 27 into numerical problems [16]. To implement the time-domain 29 Doppler filter, we can use:

- a finite impulse response (FIR) filter as the shaping filter 32 which the case in [12], [17], [20], [21],

- or an infinite impulse response (IIR) filter as in [22], [23], 35 \}; [19], [24]

The narrow-band filter with a sharp cutoff and a very large attenuation in the stop-band requires a large-order filter in the case of FIR filter [7], [24]. Meeting the same specification with an IIR filter typically requires smaller order and therefore fewer resources. However, the IIR is not necessarily stable [7] and a careful design is necessary. Due to the severe time constraints of our application, we have chosen the IIR approach. In order to closely approximate $H(f)=\sqrt{S(f)}$ in the passband while providing large suppression in the stopband, we used an elliptic IIR filter [16].

\section{Node-to-Node Link Module Implementation}

The most complex contribution is the multipath fading which is a TDF module, it requires huge memory ressources and long time of simulation. Listing 1 shows the SystemC-AMS main implementation of this module. Line 5 defines a small_scale_fading class derived from class $s c a \_t d f:$ :sca_module. Lines 7 and 8 are successively the complex input and output ports and line 9 defines velocity input port for Doppler shift computing. The small_scale_fading module uses an instance of the compute_ssf class (lines 18 and 32) in order to perform several operations such as Channel coefficions generation, IIR doppler filtering, and tapped-delayline FIR filtering. In line 22, processing() process which encapsulates the actual processing function invokes the compute method in order to apply the multipath fading process to the actual sample. The processing() member function is called by the simulation kernel as part of time-domain simulation, where each module activation advances the local module time by the assigned or derived module time step.

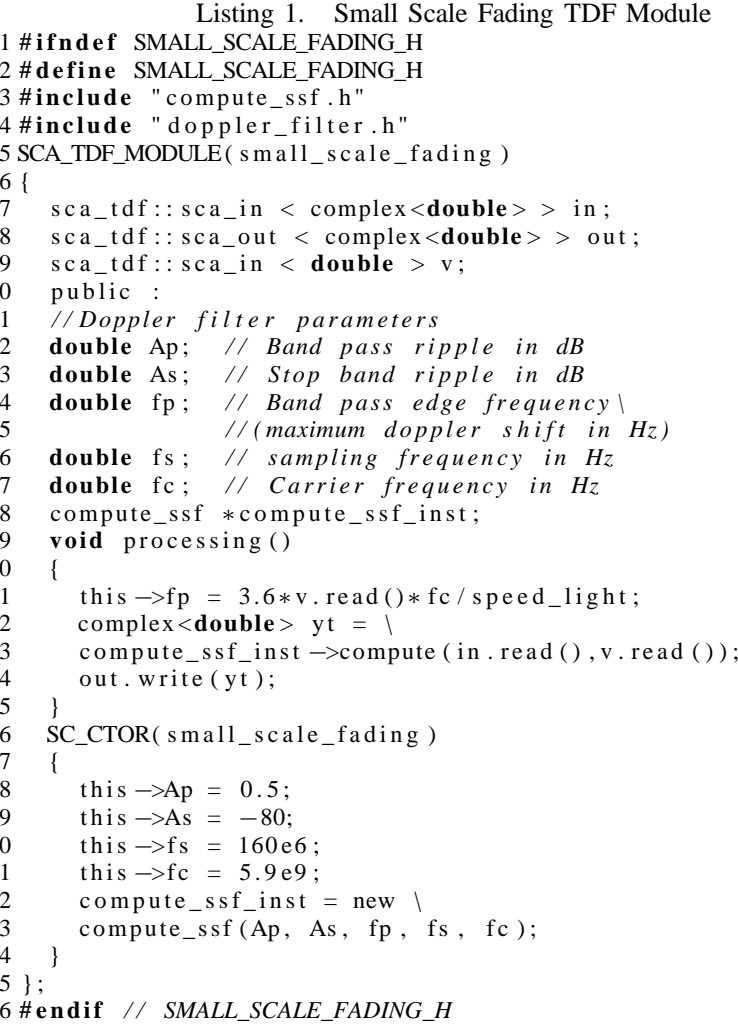

Listing 2 shows the SystemC-AMS netlist of the node-tonode link. As in listing 1 Lines 10 and 11 are successively the complex input and output ports. Line 13 defines the distance between communicating vehicles used to compute the attenuation. Line 14 defines velocity input requested by small_scale_fading module for Doppler shift computing. This netlist shows the interconnection of the three basic contributions of the considered mobile node-to-node link.

Listing 2. Node-to-Node Link Module

1 \# ifndef NODE_TO_NODE_LINK_H 


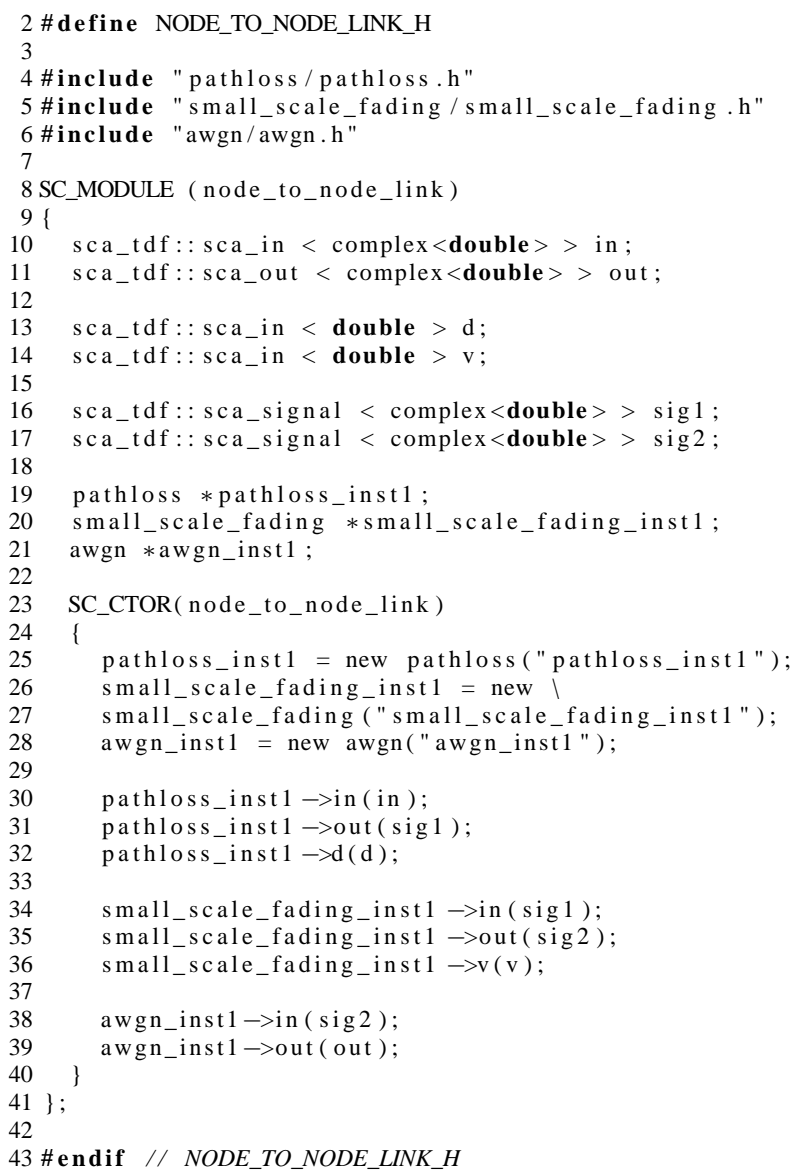

\section{RESULTS AND DISCUSSIONS}

In addition to the Analog Mixed Signal aspect, simulation speed is an important criteria of the presented framework. All simulations were run on a Linux Ubuntu machine with dual 64 bit $2.4 \mathrm{GHz}$ Intel ${ }^{\circledR}$ Xeon ${ }^{\circledR}$ processors and $12 \mathrm{~GB}$ of memory. There are two important aspects of the multipath fading phenomenon: the phase variations and the time-varying enveloppe or amplitude. The fading component will vary the signal envelope depending on fades and peaks, while the phase component will rotate the signal constellation depending of the sum of the arriving paths. The best way to describe this phenomenon is to transmit a QPSK signal and emphasize the received constellation diagram and the eye diagram for different time-varying fading. Figure 4 shows the received signal constellation (Fig. 4-c and Fig. 4-d) and the eye diagram (Fig. 4-e and Fig. 4-f) after encountering the multipath faded channel for two different speeds $(12 \mathrm{~km} / \mathrm{h}$ : Fig. $4-\mathrm{a}$ and $120 \mathrm{~km} / \mathrm{h}$ : Fig. 4-b). The most important point which should be mentioned is that when the signal encounters a fast fading (greater speed), the signal amplitude decreases, and the phase changes sharply. This causes a rotation of the signal constellation and affects the eye diagram: its opening is reduced so that the time over which we can sucessfuly sample the received signal is reduced and the resulting sample is more sensitive to noise. The complete heterogeneous communicating nodes through wireless channel were implemented and simulated using SystemC-AMS. The complexity of this application resides not only in its real and continuous time aspect but also its time and memory-consuming aspect. The transmission part (RF transceiver and wireless channel) of the 5 cars scenario is implemented and simulated using matlab and SystemC-AMS. These two different simulations were run on the same machine to compare the simulation time. We assumed that each car has 10 packets to be transmitted to the other cars using TDMA protocol. This leads on 50 Time Slots as the global time of simulation. The dependancy of the time of simulation on the length of transmitted packet and the number of multipath components (paths) is shown in figure 5. It shows that the simulation of this kind of complex scenario can be performed using SystemC-AMS in reasonable times while ensuring these fondamental aspects: (1) continuous time simulation, (2) concurrent and parallel execution of the different instantiated wireless channels and (3) oriented object aspect inhereted from $\mathrm{C}++$ which leads in an easy way of instantiating modules and a best way of memory allocation and disallocation management.

\section{CONCLUSION}

To solve the design challenges of modern wireless communication systems used in WSN due to its heterogeneous nature and its required long time of simulation not only in the circuit level but also in the system level, we have considered a baseband model of a mobile node (car) and we have simulated a 5 communicationg nodes scenario based on TDMA protocol using SystemC-AMS. This scenario which could be considered as a WSN of a small number of nodes, needs 20 time-varying wireless channels to ensure communication between nodes which makes simulation run slowly.

This paper presents the advantages of using SystemC-AMS in order to model heterogeneous systems and performing real time continuous simulations. In addition, it shows that using SystemC-AMS in complex scenario can reduce the total time of simulation compared to the actual CAD tools such as matlab. Our future work is to extend the developped toolbox using SystemC-AMS in order to ensure equalization, channel coding and channel decoding functionalities, and to provide solutions and results for the high level estimation of the consumed-power for WSN.

\section{REFERENCES}

[1] Alain Vachoux, Christoph Grimm, Karsten Einwich, "Extending SystemC to Support Mixed Discrete-Continuous System Modeling and Simulation," ISCAS 2005.

[2] http://www.systemc.org/downloads/standards/ams10/

[3] http://systemc-ams.eas.iis.fraunhofer.de

[4] Bello, P. , "Characterization of Randomly Time-Variant Linear Channels," Communications Systems, IEEE Transactions on, vol. 11, no. 4, pp. 360393, Dec 1963

[5] E. R. Golder, and J. G. Settle "The Box-M Method for Generating Pseudo-random Normal Deviates," Appl. Statist., 25, No. 1, pp. 12, 1976

[6] M. J. Gans, "A power-spectral theory of propagation in the mobile-radio environment," IEEE Trans. Veh. Technol., vol. VT-21, no. 1, pp. 27-38, Feb. 1972.

[7] M. C. Jeruchim, P. Balaban, and K. S. Shanmugan, "Simulation of Communication Systems - Modeling, Methodology, and Techniques," KluwerAcademic/Plenum Publishers, 2nd edition, 2000. 

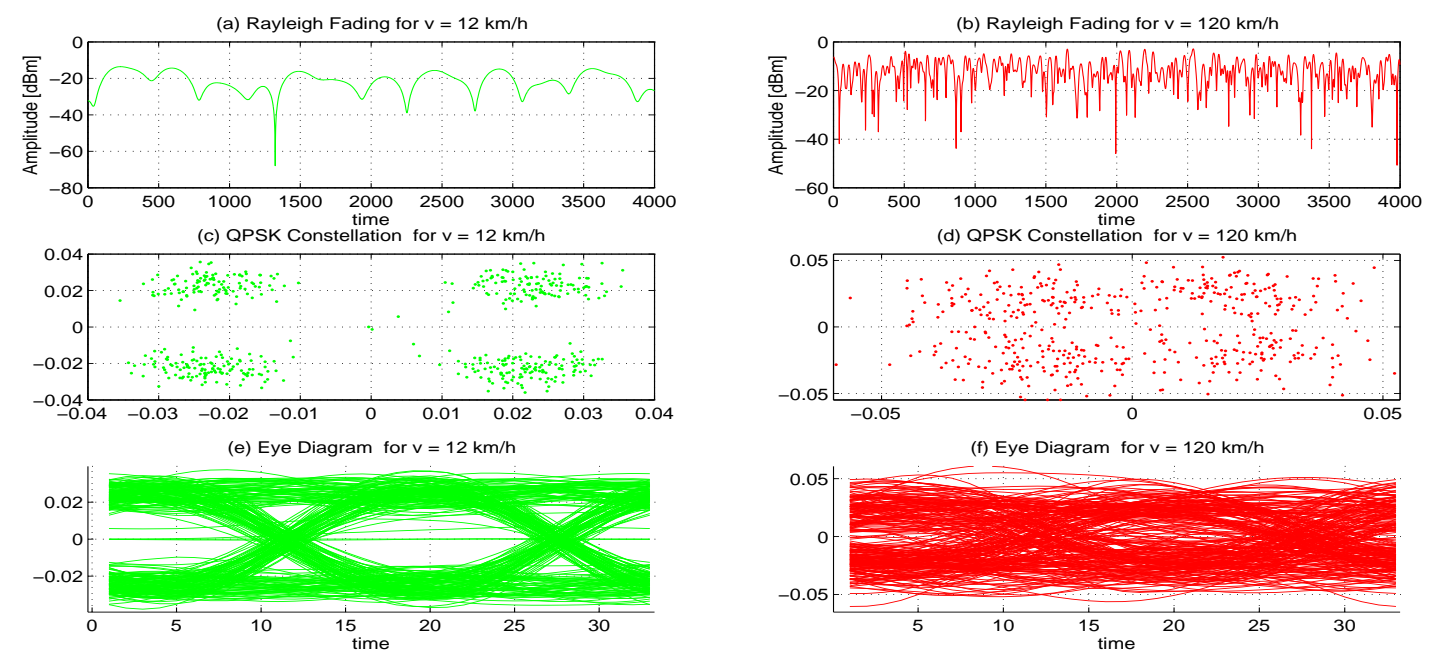

Figure 4. Evolution of the channel coefficient (Rayleigh fading) (a) @ $12 \mathrm{~km} / \mathrm{h}$ and (b) @ $120 \mathrm{~km} / \mathrm{h}$ and its impact on the QPSK constellation (c) and (d) and on the eye diagram (e) and (f).
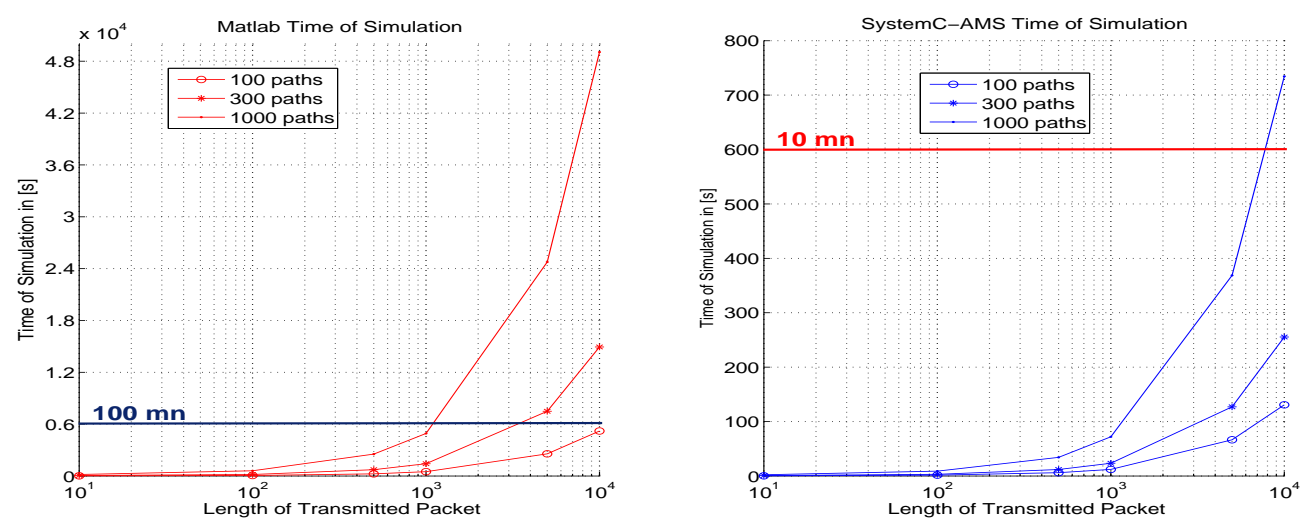

Figure 5. Comparaison of the total simulation time between Matlab and SystemC-AMS for the 5 nodes scenario using TDMA for 50 Time Slots.

[8] G. L. Turin et al., "A statistical model of urban multipath propagation," IEEE Trans. Veh. Technol., vol. VT-21, no. 1, pp. 1-9, Feb. 1972.

[9] W. C. Jakes, "Microwave Mobile Communications," IEEE Press, 1993, New York.

[10] COST 2007, "Digital land mobile radio communication" Office for Official Publications of the European Communities, Final report, Luxembourg, 1989

[11] J. G. Proakis, "Digital Communications,' McGraw-Hill, 4th edition, 2001

[12] J. I. Smith, "A computer general multipath fading simulation for mobile radio," IEEE Trans. Veh Technol., vol. VT-24, no. 3, pp. 39-40, Aug, 1975

[13] D. J. Young and N. C. Beaulieu, "The generation of correlated Rayleigh random variates by inverse discrete Fourrier transform," IEEE Trans. Commun., vol. 48, no. 7, pp. 1114-1127, Jul, 2000

[14] T. Groetker, S. Lio, G. Martin and S. Swan, "System design with systemc," Kluwer Academic Publishers, 2002.

[15] C. Komninakis, "A fast and accurate Rayleigh fading simulator" Proc IEEE Global Telecommun. Conf., 2003, pp. 3306-3310

[16] L.B. Jackson, "Digital Filters and Signal Processing." Norwell, MA: Kluer, 1989.

[17] A. Verschoor, A. Kegel, and J. C. Arnbak, "Hardware fading simulator for a number of narrowbnad channels with controllable mutual correlation," Electron. Lett., vol. 24, no. 22, pp. 1367-1369, Oct. 1988.
[18] A. K. Salkintzis, "Implementation of a digital wide-band mobile channel simulator" IEE Trans, Broadcast., vol. 45, no. 1, pp. 122-128, Mar. 1999

[19] M. A. Wickert and j. Papenfuss, "Implementation of a real-time frequency-selective RF channel simulator using a hybrid DSP-FPGA architecture," IEEE Trans. Microw. Theory Tech., vol. 49, no. 8, pp. 1390-1397, Aug. 2001

[20] R. A. Goubran, H. M. Hafez, and A. U. H. Sheikh, "Implementation of real-time mobile channel simulator using a DSP chip," IEEE Trans. Instrum. Meas., vol. 40, no. 4, pp. 709-714, Aug. 1991

[21] M. S. Lim and H. K. Park, "The implementation of the mobile channel simulator in the baseband and its application to the quadrature type GMSK modem design," Proc. IEEE Veh. Technol. Conf., 1990, pp. 469500

[22] K. E. Baddour and N. C. Beaulieu, "Autoregressive models for fading channel simulation," Proc. IEEE Global Telecommun. Conf., 2001, pp. 1187-1192

[23] M. Lecours and F. Marceau, "Design and implementation of a channel simulator for wideband mobile radio transmission," Proc. IEEE Veh. Technol. Conf., 1989, pp. 652-655

[24] A. Stéphenne and B. Champagne, "Effective multi-path vector channel simulator for antenna array systems," IEEE Trans. Veh. Technol., vol. 49, no. 6 , pp. 2370-2381, Nov. 2000 